\title{
Rare Case of a Patient with Newly Diagnosed Thymoma Presenting with Paraneoplastic Systemic Lupus Erythematosus, Myasthenia Gravis, and Hypogammaglobulinemia
}

\author{
Damien Mikael Hansra ${ }^{1}$, Mario Ponce ${ }^{2}$, Francisco Socola ${ }^{2}$, Maria Helena Restrepo ${ }^{3}$ \\ ${ }^{1}$ Department of Hematology/Oncology, Sylvester Comprehensive Cancer Institute, Jackson Memorial Hospit- \\ al/University of Miami, Miami, Florida, USA \\ ${ }^{2}$ Department of Medicine, Jackson Memorial Hospital/University of Miami, Miami, Florida, USA \\ ${ }^{3}$ Department of Hematology/Oncology, Sylvester Comprehensive Cancer Institute, University of Miami, Miami, \\ Florida, USA \\ Email: ${ }^{\text {dmhansra@med.miami.edu }}$
}

Received 20 May 2014; revised 20 June 2014; accepted 20 July 2014

Copyright (C) 2014 by authors and Scientific Research Publishing Inc.

This work is licensed under the Creative Commons Attribution International License (CC BY). http://creativecommons.org/licenses/by/4.0/

(c) (i) Open Access

\begin{abstract}
A Pleural mass biopsy was performed showing an invasive thymoma. Computed tomography (CT) scan of the chest performed on admission revealed a left sided pleural mass, anterior mediastinal mass, lymphadenopathy and pericardial effusion. Pleural mass biopsy showed an invasive thymoma. Due to her clinical presentation, a complete work-up was performed revealing paraneoplastic systemic lupus erythematous (SLE), myasthenia gravis (MG) and hypogammaglobulinemia. We reviewed the literature regarding thymoma and its relationship with paraneoplastic SLE, MG and hypogammaglobulinemia.
\end{abstract}

\section{Keywords}

Thymoma, Paraneoplastic, Systemic Lupus Erythematosus, Myasthenia Gravis, Hypogammaglobulinemia

\section{Introduction}

Thymomas are associated with a wide variety of paraneoplastic disorders. Paraneoplastic syndromes are present in over $50 \%$ of patients and more than one syndrome may be present. Here we describe a rare case of a patient "Corresponding author.

How to cite this paper: Hansra, D.M., et al. (2014) Rare Case of a Patient with Newly Diagnosed Thymoma Presenting with Paraneoplastic Systemic Lupus Erythematosus, Myasthenia Gravis, and Hypogammaglobulinemia. Open Journal of Rheumatology and Autoimmune Diseases, 4, 146-152. http://dx.doi.org/10.4236/ojra.2014.43021 
with newly diagnosed thymoma presenting with MG, SLE, and hypogammaglobulinemia. Also, we have provided a discussion of key diagnostic and epidemiologic features of these paraneoplastic entities.

\section{Case Report}

A 53-year-old female with no past medical history arrives at the emergency room for one month of fevers, malaise, productive cough, and thirty pound weight loss, associated with gradually increasing progressive shortness of breath, dyspnea on exertion, and diplopia worse at end of day. She previously received treatment for pneumonia at an outside facility a month earlier with transient improvement in fevers and cough however she experienced progressive symptoms. Initial chest $\mathrm{x}$-ray showed nodular opacities projecting over left mid and lower lung zones. CT chest (Figure 1): multiple left sided pleural based masses, anterior mediastinal mass $4.7 \times 1.6$ $\mathrm{cm}$, right sided axillary lymphadenopathy, and small pericardial effusion. The patient was started on empiric intravenous antibiotics for community acquired pneumonia and admitted to medical service for further workup.

Cardiothoracic surgery was consulted and performed fiberoptic bronchoscopy, left thoracoscopy, biopsy of pleural-based masses, pericardial window with biopsy and fluid analysis, and intraoperative transesophageal echocardiogram (TEE). TEE showed moderate pericardial effusion with compression of right atrium and depressed EF 45\%, no focal wall motion abnormality identified. On thoracoscopy, there were multiple pleural, diaphragmatic and pericardial based masses. Pericardial window was performed, which drained bloody pericardial fluid. Cytology on fluid was negative. Pleural based mass biopsy revealed invasive thymoma. The pericardial biopsy was negative.

Histologically, the tumor cells form lobules that are separated by thick fibrous and hyalinized septa (Figure 2). The tumor invades the pleura. The tumor is predominantly composed of medium sized round or polygonal cells with slight atypia admixed with few intraepithelial lymphocytes resulting in the formation of tumor cell sheets with a vaguely solid and epidermoid appearance (Figure 3). No necrosis, mitotic activity, or features of thymic carcinoma were observed. The tumor cells were positive for keratin and P63 and negative for CD5, TTF-1, CEA, D2-40, S100 and B72.3 (Figure 4 and Figure 5). The patient was staged as T4N0M0 stage IV, WHO Type B3, invasive thymoma.

Clinically, the patient was found patient to have fatigability with repetitive extremity use, ptosis, and diplopia. The patient was empirically started on pyridostigmine and prednisone for paraneoplastic MG with improvement in symptoms within days. Electromyography (EMG) demonstrated dysfunction in myoneural junction transmission. Antibody panel was sent and the patient had positive acetylcholine receptor antibodies $(161 \mathrm{nmol} / \mathrm{L})$, acetylcholine receptor modulating antibodies $(94 \mathrm{nmol} / \mathrm{L}$ ), and acetylcholine receptor blocking antibodies (45 $\mathrm{nmol} / \mathrm{L}$ ). With the constellation of clinical, nerve conduction studies, and antibody assays the patient was confirmed to have paraneoplastic MG.

Rheumatology service consulted for patient's oral ulcerations, cytology negative pericardial effusion (Figure 6), and constitutional symptomswhich developed around initial presentation. There were obvious oral ulcera-

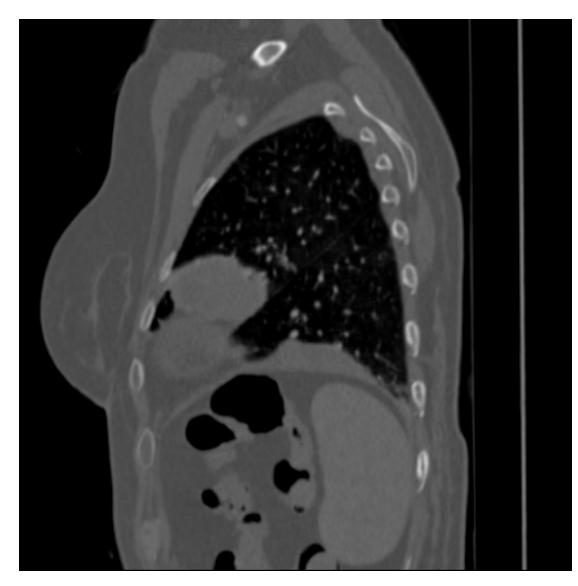

Figure 1. CT chest demonstrating anterior mediastinal mass and pleural based masses. 


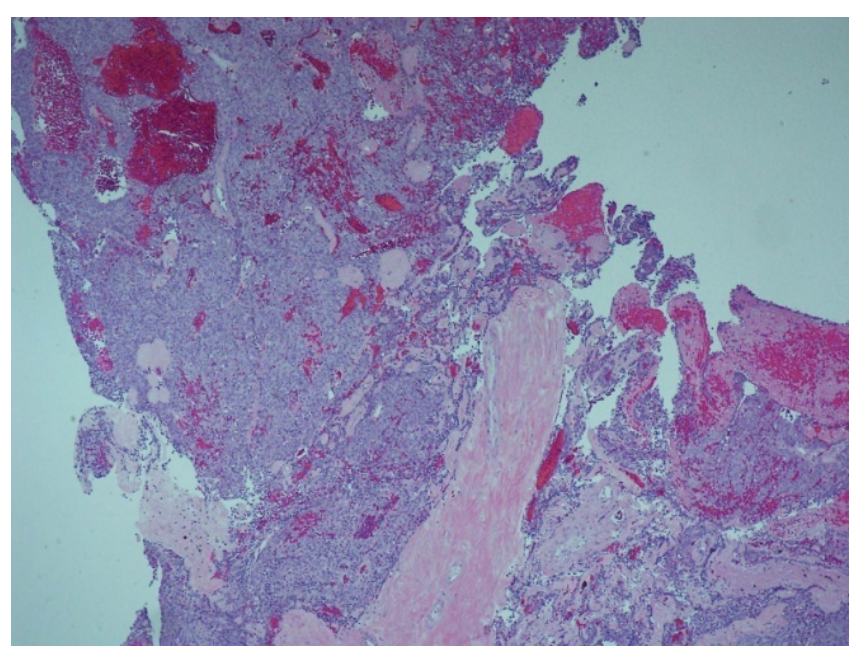

Figure 2. Tumor forming lobules which are separated by fibrous septa.

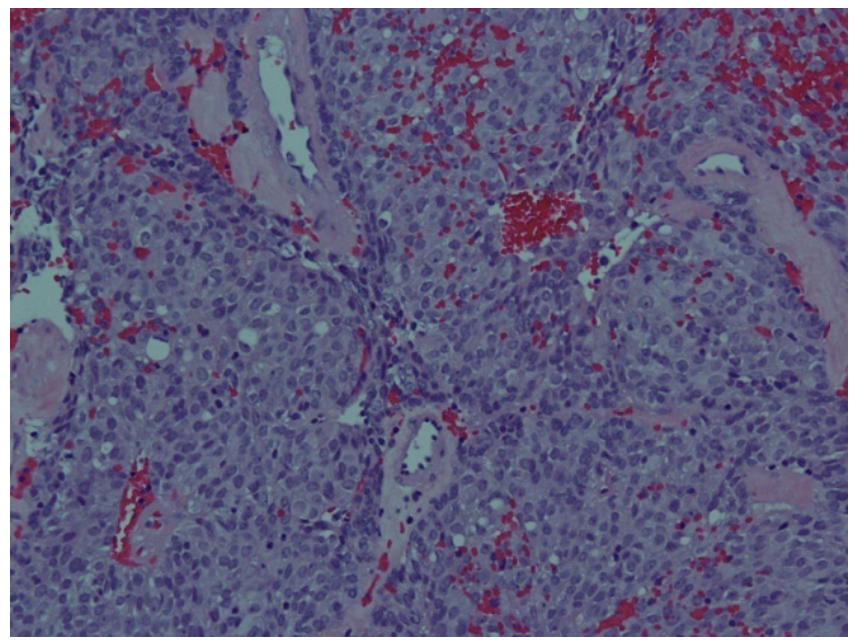

Figure 3. Tumor cells are polygonal in shape and few scattered lymphocytes.

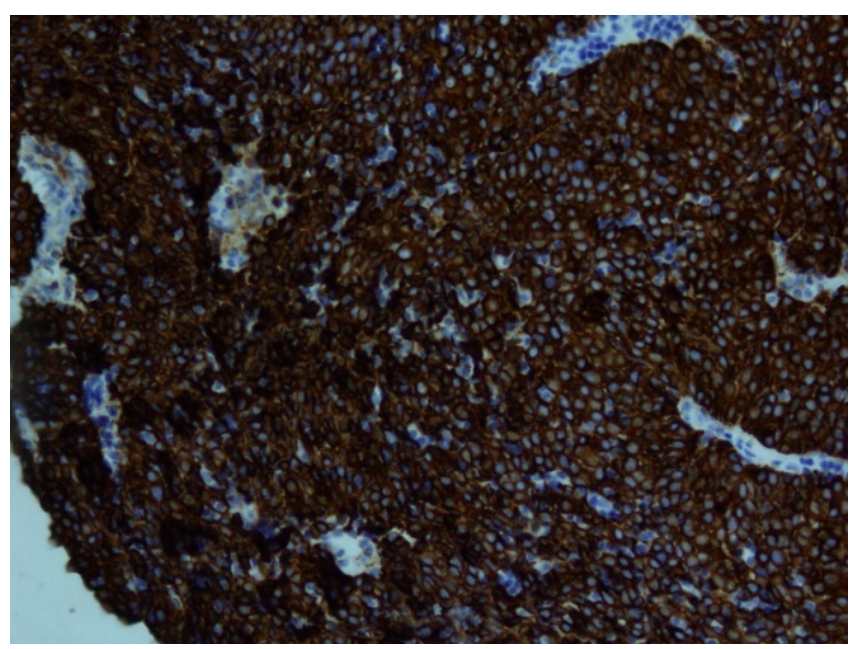

Figure 4. Diffuse positivity for keratin. 


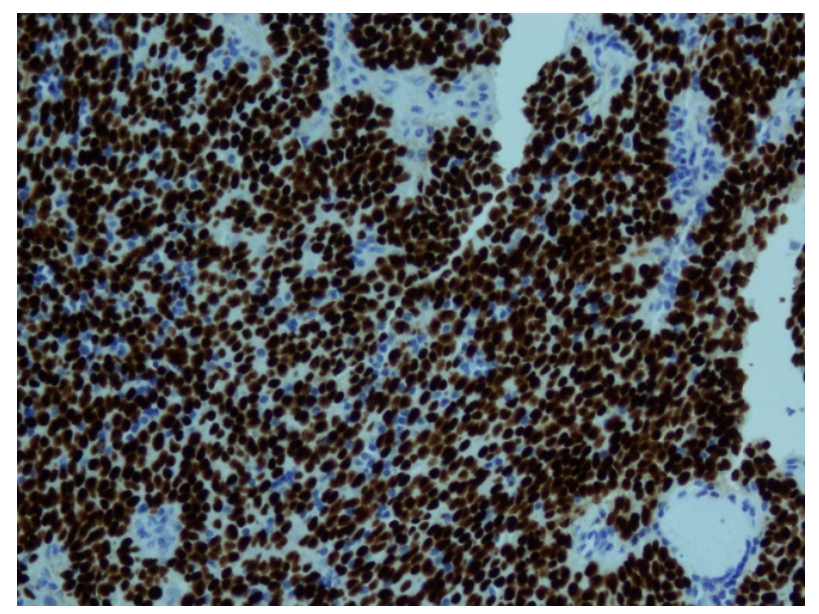

Figure 5. Diffuse positivity for P63 immunostain.

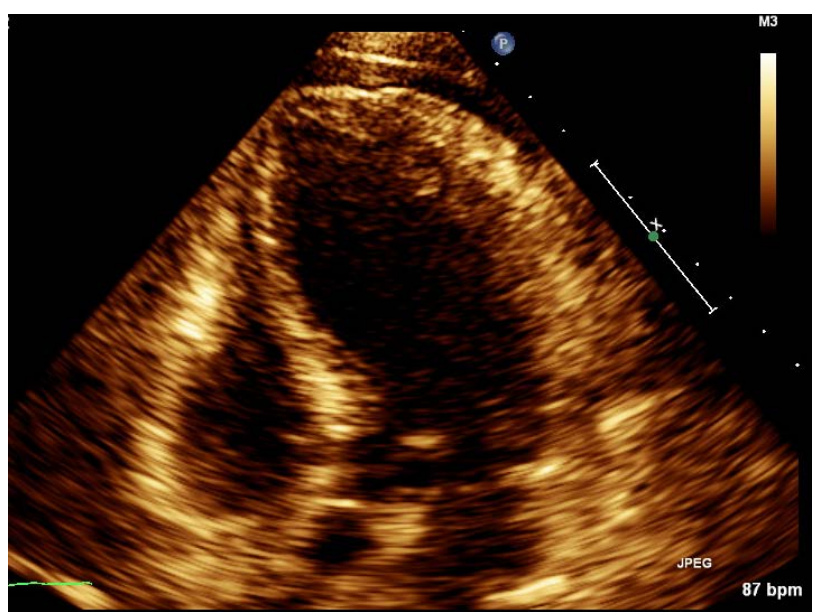

Figure 6. 2D echo demonstrating moderate pleural effusion.

tions at the lateral aspect of the tongue that were bright red, elevated. Biopsy demonstrated squamous mucosa with marked keratosis and band-like lymphplasmacytic inflammatory infiltrate in the submucosa. Also, the patient demonstrated high antinuclear antibody titer (2560), high anti-double stranded DNA (43 IU/ml), low complement level C3 (62.5 mg/dl) and low C4 (5 mg/dl). Also, anti-chromatin antibodies were positive. Based on clinical and laboratory symptoms the patient was diagnosed with paraneoplastic SLE.

The patient was also found to have hypogammaglobulinemia during admission. Serum protein electrophoresis demonstrated non-specific hypogammaglobulinemia, serum gamma globulin fraction was low (0.50 g/dL), serum beta globulin fraction was low $(0.42 \mathrm{~g} / \mathrm{dL})$, and serum alpha fractions were normal. Serum IgG was low (526 mg/dL), serum IgA was low normal (88.20 mg/dL), serum IgM was low normal IgM $58.2 \mathrm{mg} / \mathrm{dL}$ (low normal), Kappa light chains were low $(114.00 \mathrm{mg} / \mathrm{dL})$, and Lambda light chains were low (83.50 mg/dL). The patient had a normal CD4+ count of 1006 cells/mcl and a normal CD4/CD8 ratio of 2.17. HIV test was negative. The Patient was diagnosed with mild paraneoplastic hypogammaglobulinemia based on laboratory findings.

\section{Discussion}

Thymomas are associated with a wide variety of paraneoplastic disorders, the most common of which is MG [1]. Paraneoplastic syndromes are present in over 50\% of patients and more than one syndrome may be present. Here we describe a rare case of a patient with newly diagnosed thymoma presenting with MG, SLE, and hypogammaglobulinemia. Also, we have provided a discussion of key diagnostic and epidemiologic features of these paraneoplastic entities. 


\subsection{Myasthenia Gravis}

MG is a neuromuscular junction disorder caused by the autoimmune interference with acetylcholine receptors of voluntary muscle. Symptoms include diplopia, ptosis, dysphagia, weakness and fatigue. In patients with thymoma, $40 \%$ have one or more paraneoplastic autoimmune conditions, about $20 \%-25 \%$ of which is MG [2]. Conversely, $10 \%-15 \%$ of patients with MG are observed to have thymoma. Of myasthenia patients with thymoma, 4\% - 7\% have more than one paraneoplastic syndrome [2] [3]. About $10 \%-15 \%$ of patients with thymoma have paraneoplastic conditions other than MG [4]. Women are twice affected as often as men. The major feature of MG is fluctuation of fatigable weakness. The presenting symptoms are ocular in half of patients in which half of patients present with diplopia and the other half with ptosis [5]. Other prominent presenting symptoms are bulbar (dysarthria or dysphagia) in 10\%, leg weakness in 10\% and generalized weakness in $10 \%$ [5]. The diagnosis of MG is based on history of fluctuating weakness with corroborating findings on examination. There are many ways to confirm the clinical diagnosis such as edrophonium testing, antibody assays, and electrophysiologic testing. The most immediate and readily accessible confirmatory study is the edrophonium (Tensilon) test [6]. The sensitivity of the test is about $90 \%$. The specificity is difficult to determine, as improvement following edrophonium has been reported in other neuromuscular diseases, including Lambert Eaton syndrome, botulism, Guillan-Barre syndrome, motor neuron disease, and lesions of the brainstem and cavernous sinus. The standard assay for receptor binding antibodies is an immunoprecipitation assay using human limb muscle for acetylcholine receptor antigen. In addition, assays for receptor modulating and blocking antibodies are available. Binding antibodies are present in about $80 \%$ of all MG patients [7]. By also testing for modulating and blocking antibodies, the sensitivity improves to $90 \%$ overall. The specificity is $>95 \%$ and false positive tests are extremely rare [7]. Electrophysiologic testing is a widely available test with a sensitivity of approximately $50 \%$. The yield from repetitive stimulation is higher when testing muscle groups have significant clinical weakness. Single fiber EMG is a highly specialized technique with less availability with a sensitivity of $90 \%$ [8]. Our patient presented with ptosis and fluctuating weakness most prominent in the upper extremities which was consistent with MG. Repetitive nerve stimulation in upper extremity showed decremented response characteristic of MG. Additionally our patient had positive acetylcholine receptor binding, blocking, and modulating antibodies confirming the diagnosis of MG.

\subsection{Systemic Lupus Erythematosus}

The association between SLE and a neoplastic disease is rare and the relation between these two pathologies is uncertain [9]. The estimated prevalence of SLE among patients with thymoma varied between $1.5 \%$ and $2 \%$ in clinical studies [10] and between $6 \%$ and $10 \%$ in studies in which clinical data of biopsy proven thymomas were reviewed retrospectively [11] [12]. In a recent review of thymoma patients diagnosed from 1922-1998, 94\% were female, the mean age at detection of the tumor was 53 years (range 24 - 68) and the mean age at diagnosis of SLE was 52 years (range 25 - 76) [13]. In 39\% of the patients SLE had been diagnosed before detection of the thymoma (mean interval 2.9 years) [13], in 33\% thymoma and SLE were found simultaneously and in 28\% SLE had been diagnosed after discovery of the thymoma (mean interval 2.7 years) [13]. Clinically, the most commonly reported symptoms were arthralgia or arthritis in 58\%, skin involvement in $25 \%$ and pleuritis or pericarditis in 63\% [13]. Autoimmune serology was positive in all cases [13]. Three patients had concurrent MG and one patient had pure red cell aplasia. In the cases with histological data, the most common histological type of thymoma was mixed lymphoepithelial (60\%), whereas in $47 \%$ of cases it was invasive [13]. Our patient presented in similar demographic as the reported literature: female and early fifties. She was diagnosed based on oral ulcerations, cytology negative pericardial effusion, and constitutional symptoms which developed around initial presentation. Most convincingly, the patient demonstrated high antinuclear antibody titer (2560), high anti-double stranded DNA (43 IU/ml), low complement level C3 (62.5 mg/dl) and low C4 (5 mg/dl), and antichromatin antibodies were positive. Therefore with the constellation of clinical and laboratory findings, the patient was diagnosed with paraneoplastic lupus.

\subsection{Hypogammaglobulinemia}

Hypogammaglobulinemia is present in less than 5 percent of patients with thymoma, occurring most commonly in older women [14]. Good Syndrome (GS) refers to a rare constellation of thymoma and adult-onset immunodeficiency that is characterized by low or absent B cells in the peripheral blood, hypogammaglobulinemia, and 
variable defects in cell-mediated immunity with CD4+ T-lymphopenia and an inverted CD4:CD8+ T-cell ratio. [15]. In a review of 51 patients with GS, the infections reported included recurrent sinopulmonary infections (19 cases with documented respiratory pathogens), generally with encapsulated bacteria, most often Haemophilus influenzae (11 cases); CMV disease (5 cases); bacteremia (7 cases); oral or esophageal candidiasis (6 cases); persistent mucocutaneous candidiasis (5 cases); chronic diarrhea (5 cases with documented stool pathogens); urinary tract infections (4 cases); P. carinii pneumonia (3 cases); tuberculosis (2 cases); Kaposi sarcoma (1 case); disseminated varicella (1 case); candidemia (1 case); wound infection with Clostridium perfringens (1 case); Mycoplasma arthritis (1 case); and other infections [15]. Male and female patients were about equally affected. Patients were most often in their fourth or fifth decade when they developed thymoma. Patients were commonly diagnosed with thymoma upon first infectious presentation of their immunodeficiency [15]. Our patient presented with recurrent pulmonary infections however no definite pathogen was identified on blood or sputum cultures. The patient was diagnosed with mild paraneoplastic hypogammaglobulinemia based on recurrent infections and serum studies. Patient did not however meet strict criteria for GS given normal lymphocyte count on peripheral analysis and preserved CD4+ numbers and CD4/DC8+ ratio.

Thymomas are associated with a wide variety of paraneoplastic disorders. Paraneoplastic syndromes are present in over $50 \%$ of patients and more than one syndrome may be present. Here we have described a rare case of a patient with newly diagnosed thymoma presenting with MG, SLE, and hypogammaglobulinemia. This case is important as it highlights three of many potential paraneoplastic syndromes occurring in thymoma. Furthermore, the clinician must maintain a steady awareness and a high index of suspicion for paraneoplastic disorders in this oncologic population as these syndromes may cause significant morbidity and thus offer potential targets for clinical intervention.

\section{References}

[1] Tomoehlen, L.M. and Pascuzzi, R.M. (2008) Thymoma, Myasthenia Gravis, and Other Paraneoplastic Syndromes. Hematology/Oncology Clinics of North America, 22, 509-526 http://dx.doi.org/10.1016/j.hoc.2008.03.004

[2] Lewis, J.E., Wick, M.R., Scheithauer, B.W., Bernatz, P.E. and Taylor, W.F. (1987) Thymoma. A Clinicopathologic Review. Cancer, 60, 2727-2743. http://dx.doi.org/10.1002/1097-0142(19871201)60:11<2727::AID-CNCR2820601125>3.0.CO;2-D

[3] Monden, Y., Uyama, T., Nakahara, K., et al. (1986) Clinical Characteristic and Prognosis of Myasthenia Gravis with Other Autoimmune Diseases. The Annals of Thoracic Surgery, 41, 189-192. http://dx.doi.org/10.1016/S0003-4975(10)62667-7

[4] Evoli, A., Minicuci, G.M., Vitaliani, R., et al. (2007) Paraneoplastic Diseases Associated with Thymoma. Journal of Neurology, 254, 756-762. http://dx.doi.org/10.1007/s00415-006-0429-Z

[5] Grob, D., Arsura, E.L., Brunner, N.G., et al. (1987) The Course of Myasthenia Gravis and Therapies Affecting Outcome. Annals of the New York Academy of Sciences, 505, 472-499. http://dx.doi.org/10.1111/j.1749-6632.1987.tb51317.x

[6] Pascuzzi, R.M. (2003) The Edrophonium Test. Seminars in Neurology, 23, 83-88. http://dx.doi.org/10.1055/s-2003-40755

[7] Howard, F.M., Lenin, V.A., Finley, J., et al. (1987) Clinical Correlations of Antibodies That Bind, Block, or Modulate Human Acetylcholine Receptors in Myasthenia Gravis. Annals of the New York Academy of Sciences, 505, 526-538. http://dx.doi.org/10.1111/j.1749-6632.1987.tb51321.x

[8] Pascuzzi, R.M. (2001) Pearls and Pitfalls in the Diagnosis and Management of Neuromuscular Junction Disorders. Seminars in Neurology, 21, 425-440. http://dx.doi.org/10.1055/s-2001-19414

[9] Chakravarty, E. and Genovese, M.C. (2003) Rheumatic Syndromes Associated with Malignancy. Current Opinion in Rheumatology, 15, 35-43. http://dx.doi.org/10.1097/00002281-200301000-00007

[10] Mevorach, D., Perrot, S., Buchanan, N.M., Khamashta, M., Laoussadi, S., Hughes, G.R., et al. (1995) Appearance of Systemic Lupus Erythematosus after Thymectomy: Four Case Reports and Review of the Literature. Lupus, 4, 33-37. http://dx.doi.org/10.1177/096120339500400108

[11] Oosterhuis, H.J., Feltkamp, T.E., van Rossum, A.L., van den Berg-Loonen, P.M. and Nijenhuis, L.E. (1976) HL-A Antigens, Autoantibody Production, and Associated Diseases in Thymoma Patients, with and without Myasthenia Gravis. Annals of the New York Academy of Sciences, 274, 468-474. http://dx.doi.org/10.1111/j.1749-6632.1976.tb47708.x

[12] Steven, M.M., Westedt, M.L., Eulderink, F., Hazevoet, H.M., Dijkman, J.H. and Cats, A. (1984) Systemic Lupus Erythematosus and Invasive Thymoma: Report of Two Cases. Annals of the Rheumatic Diseases, 43, 825-828. 
http://dx.doi.org/10.1136/ard.43.6.825

[13] Boonen, A., Rennenberg, R. and van der Linden, S. (2000) Thymoma-Associated Systemic Lupus Erythematosus, Exacerbating after Thymectomy. A Case Report and Review of the Literature. Rheumatology, 39, 1044-1046 http://dx.doi.org/10.1093/rheumatology/39.9.1044

[14] Verley, J.M. and Hollmann, K.H. (1985) Thymoma. A Comparative Study of Clinical Stages, Histologic Features, and Survival in 200 Cases. Cancer, 55, 1074-1086. http://dx.doi.org/10.1002/1097-0142(19850301)55:5<1074::AID-CNCR2820550524>3.0.CO;2-T

[15] Tarr, P.E., Sneller, M.C., Mechanic, L.J., et al. (2001) Infections in Patients with Immunodeficiency with Thymoma (Good Syndrome). Report of 5 Cases and Review of the Literature. Medicine (Baltimore), 80, 123-133. http://dx.doi.org/10.1097/00005792-200103000-00005 
Scientific Research Publishing (SCIRP) is one of the largest Open Access journal publishers. It is currently publishing more than 200 open access, online, peer-reviewed journals covering a wide range of academic disciplines. SCIRP serves the worldwide academic communities and contributes to the progress and application of science with its publication.

Other selected journals from SCIRP are listed as below. Submit your manuscript to us via either submit@scirp.org or Online Submission Portal.
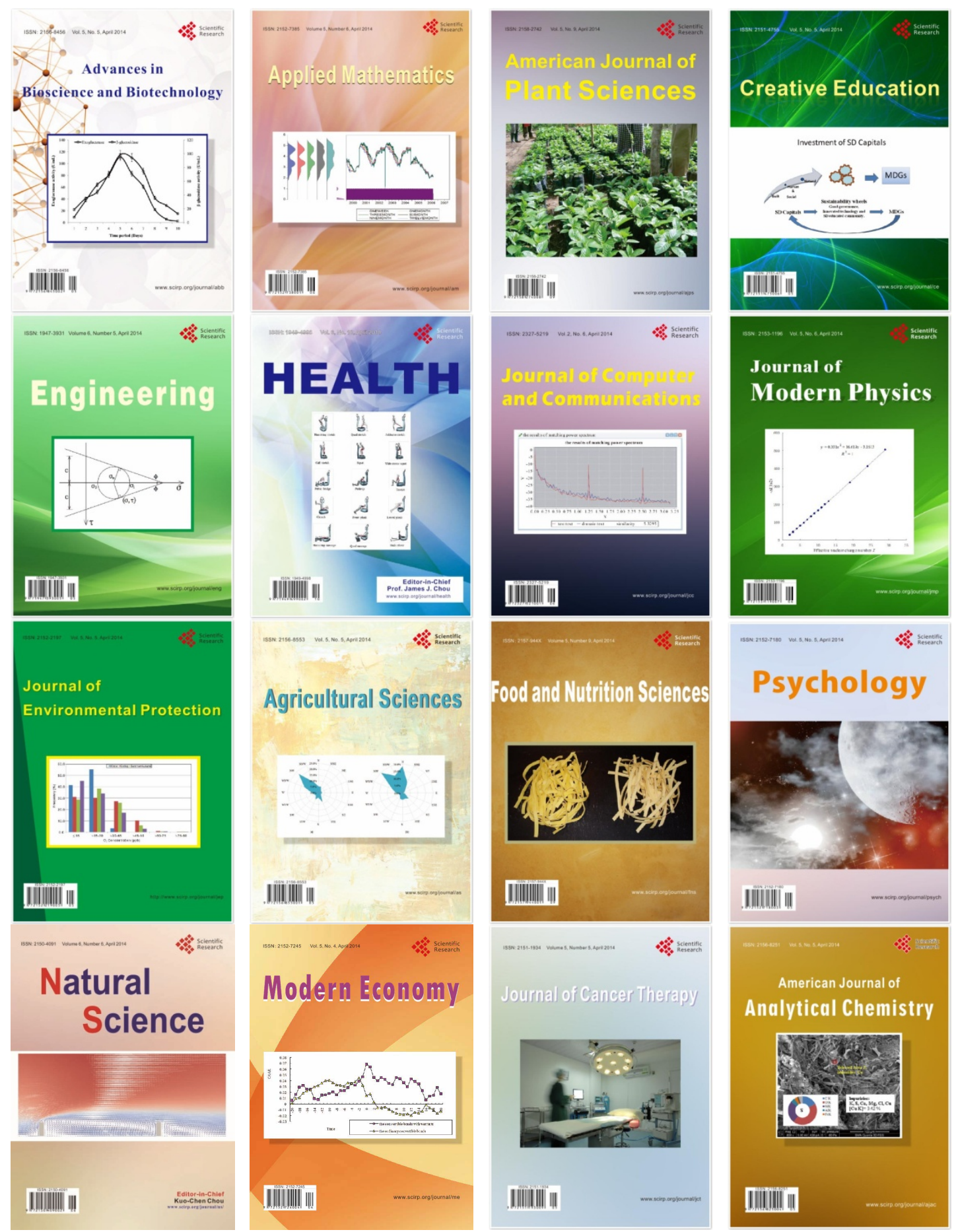